\title{
The average behaviour of greedy algorithms for the knapsack problem: General distributions. I
}

\author{
Gennady Diubin and Alexander Korbut*
}

\begin{abstract}
This paper is a partial generalization of the results of [3] for rather arbitrary distributions of coefficients. We state the main theorem concerning the average behaviour of greedy algorithms. The validity of this theorem is implied by the validity of the Conditions 1 and 2 from [3]. We give a detailed proof of Condition 1. The characterization of distributions for which Condition 2 holds will be the subject of a forthcoming paper.
\end{abstract}

\section{Introduction}

Our main object is the classical knapsack problem with Boolean variables. It consists in finding

$$
f^{*}=\max \left\{\sum_{j=1}^{n} c_{j} x_{j} \mid \sum_{j=1}^{n} a_{j} x_{j} \leq b, x \in \mathbb{B}^{n}\right\} .
$$

All coefficients in (1) are positive. The standard interpretation of the problem (1) is the following: We have to fill a knapsack of capacity $b$ with the most profitable subset of items from $\{1, \ldots, n\}$, where each item $j$ is characterized by its utility $c_{j}$ and weight $a_{j}$. The Boolean variables $x_{j}$ equal 1 if the item $j$ is chosen, and 0 otherwise.

Without loss of generality, we can suppose that $a_{j}<b$ for all $j$ and that $\sum_{j=1}^{n} a_{j}>b$. Moreover, we shall suppose that

$$
\frac{c_{1}}{a_{1}} \geq \frac{c_{2}}{a_{2}} \geq \cdots \geq \frac{c_{n}}{a_{n}}
$$

i.e., the variables $x_{j}$ are numbered in the non-increasing order of their "weight densities" $c_{j} / a_{j}$. The condition (2) is often called the regularity condition.

The problen (1) has numerous applications, and it is one of the main models of combinatorial optimization. From the point of view of the general complexity theory, it is $N P$-hard. This means that exact algorithms with polynomial complexity can only exist

\footnotetext{
${ }^{*}$ Institute for Economics and Mathematics, Russian Academy of Sciences. Tschaikovski Str. 1, 191187 St.Petersburg, Russia. E-mail: diubin@emi.spb.su, korbut@emi.spb.su. This research was partially supported by the DFG (Grant 436 RUS 17/30/00) during the second author's stay at the Humboldt-Universität zu Berlin in autumn 2000 .
} 
in the case $P=N P$. Therefore, the main research efforts are now concentrated around approximate methods for the problem (1), and this tendency is characteristic for the entire combinatorial optimization.

Among these approximate methods, the so-called greedy methods play a major role. They can be interpreted as discrete analogues of gradient (or steepest-ascent) methods in continuous optimization. Their undoubted advantage is that for the problem (1) they work in linear time (if the regularity condition (2) is fulfilled). The greedy methods do not guarantee optimality; however, theoretical estimations of their worst-case performance can be given. Details can be found in the review paper [1].

The idea of the greedy algorithm for the problem (1) consists in a consecutive selection of items with the largest weight density $c_{j} / a_{j}$ as long as the knapsack capacity admits this. More formally, the algorithm starts with a feasible solution $x=(0, \ldots, 0)$ and consecutively replaces zeros by ones in the order of decreasing ratios $c_{j} / a_{j}$ (i.e., from the left to the right); the feasibility of the corresponding solution is checked each time. The process terminates after obtaining the last feasible solution. This solution $x^{G}$ is called the greedy solution; the corresponding objective function value is denoted by $f^{G}$.

An idea that is in some sense opposite consists in a consecutive rejection of the least profitable items (again, in the sense of the ratios $c_{j} / a_{j}$ ) until the remaining ones fit in the knapsack. In accordance with the usual terminology, such algorithms can be called dual algorithms. Therefore, the greedy algorithm described above will sometimes be called primal. More formally, the dual greedy algorithm starts with an infeasible solution $x=(1, \ldots, 1)$ and consecutively replaces ones by zeroes in the order of increasing ratios $c_{j} / a_{j}$ (i.e., from the right to the left). The feasibility of the current solution is checked each time. The process terminates when the first feasible solution is obtained. This solution $x^{D G}$ is called the dual greedy solution; the corresponding objective function value is denoted by $f^{D G}$.

First of all we note that the primal and the dual greedy solutions can be different (even if all inequalities in the regularity condition (2) are strict). The simplest example is

$$
\max \left\{6 x_{1}+4 x_{2}+x_{3} \mid 2 x_{1}+2 x_{2}+x_{3} \leq 3, x \in \mathbb{B}^{3}\right\} .
$$

Here we have $x^{G}=(1,0,1), x^{D G}=(1,0,0)$.

Up to now, practically no attention to the analysis of dual greedy algorithms was paid. The reason was probably the following "folklore theorem".

Proposition 1 The dual greedy algorithm for the problem (1) can be arbitrarily bad.

It is natural to estimate the performance of the dual greedy algorithm by the ratio $R_{D G}=f^{D G} / f^{*}$. The assertion means that $R_{D G}$ can take arbitrarily small values. To prove this, we consider the following one-parametric family of instances of (1):

$$
\max \left\{3 x_{1}+2 \lambda x_{2} \mid x_{1}+\lambda x_{2} \leq \lambda, x \in \mathbb{B}^{2}\right\},
$$

where $\lambda>3 / 2$. We have $x^{*}=(0,1)$ and $f^{*}=2 \lambda$. At the same time, $x^{D G}=(1,0)$, $f^{D G}=3$. Thus, $R_{D G}=3 / 2 \lambda$ tends to zero when $\lambda \rightarrow \infty$. 
We discuss briefly some connections between primal and dual greedy solutions. To this end, we shall need several definitions. For any vector $x \in \mathbb{B}^{n}$, we call any set of its consecutive components equal to 1 its fragment. A fragment is maximal if it cannot be extended to the left and to the right. There is a natural ordering of maximal fragments. Vectors having a unique maximal fragment are called connected. Consider now vectors from $\mathbb{B}^{n}$ satisfying one of the conditions

$$
\begin{aligned}
& x_{1} \geq x_{2} \geq \cdots \geq x_{n}, \\
& x_{1} \leq x_{2} \leq \cdots \leq x_{n} .
\end{aligned}
$$

It is clear that such vectors either do not contain any fragments (i.e., they are null vectors) or they have exactly one maximal fragment, that is, they are connected. We call vectors satisfying (5) lower connected, and vectors satisfying (6) upper connected. Thus, lower connected vectors have the form $(1, \ldots, 1,0, \ldots, 0)$, and upper connected vectors - the form $(0, \ldots, 0,1, \ldots 1)$.

Now we consider again the problem (1). We note that the primal greedy solution is not necessarily connected (cf. the example (3)). The following assertion holds.

Proposition 2 Let $x^{G}, x^{D G}$ be the primal and the dual greedy solutions to the problem (1), respectively. Then

1) $x^{D G}$ is lower connected;

2) $x^{D G} \leq x^{G}$ (the inequality is componentwise) where the equality takes place if and only if $x^{G}$ is lower connected;

3) $x^{D G}$ contains the first maximal fragment of $x^{G}$ and only this fragment.

From the assertion 2) and the positivity of the objective function coefficients it follows that $f^{D G} \leq f^{G}$. In other words, the dual greedy algorithm cannot be better than the primal one. We formulate the last assertion in a somewhat extended form, which will be important later. Consider the linear relaxation of (1), which consists in finding

$$
f^{L R}=\max \left\{\sum_{j=1}^{n} c_{j} x_{j} \mid \sum_{j=1}^{n} a_{j} x_{j} \leq b, 0 \leq x_{j} \leq 1, j=1, \ldots, n\right\} .
$$

Corollary. The following inequalities hold

$$
f^{D G} \leq f^{G} \leq f^{*} \leq f^{L R}
$$

It is well-known that the optimal solution $x^{L R}$ to the linear relaxation (7) has the form

$$
x^{L R}=\left(x_{1}, \ldots, x_{k-1}, x_{k}, 0, \ldots, 0\right),
$$

where $x_{1}=\cdots=x_{k-1}=1,0 \leq x_{k}<1$, and the index $k$ is determined as

$$
k=\max \left\{s \mid \sum_{j=1}^{s-1} a_{j} \leq b\right\}
$$


or, equivalently, as

$$
k=\min \left\{s \mid \sum_{j=1}^{s} a_{j}>b\right\} .
$$

The number $k$ is called the critical index for the problem (1). Its definition implies that

$$
\sum_{j=1}^{k-1} a_{j} \leq b, \quad \sum_{j=1}^{k} a_{j}>b .
$$

In another terminology, this means that $k$ is the first index "skipped" by the primal greedy algorithm, or, equivalently, that $\left(x_{1}, \ldots, x_{k-1}\right)$ is the first maximal fragment of the vector $x^{G}$ (or, according to the assertion 3) of Proposition 2, the unique maximal fragment of $\left.x^{D G}\right)$. The value of $x_{k}$ can be easily found explicitly:

$$
x_{k}=\frac{b-\sum_{j=1}^{k-1} a_{j}}{a_{k}} .
$$

Thus we have

$$
x^{L R}=\left(F, x_{k}, 0, \ldots, 0\right),
$$

where $F$ is the maximal fragment of $x^{D G}, 0 \leq x_{k}<1$. From this it follows immediately that

$$
f^{L R}-f^{D G}=c_{k} x_{k},
$$

where $k$ is the critical index, and $x_{k}$ is determined by (9).

The proofs together with a generalization of these results to more general combinatorial structures (independence systems) can be found in [2], [4].

We have shown above that the dual greedy algorithm can be arbitrarily bad. On the other hand, this algorithm was successfully used (as a subroutine) for solving some applied large-scale problems, where it provided remarkably good results. This controversy between bad theoretical and good actual performance of an algorithm can be, as usually, resolved by studying not the worst-case but the average behaviour of this algorithm.

We began to implement this general program in [2] (see also the preliminary publication [5]). Roughly speaking, it was shown there that if, for a random set of coefficients, the "feasibility probability" tends to 1 with the growth of the number of variables, then the objective function values for the primal and the dual greedy algorithms, with large probability, differ insignificantly from the optimal value. However, this result had in a certain sense a conditional character. It remained to find bounds for the right-hand side of the problem for which this theorem is non-trivially true (see some short comments concluding [2]).

This was done in [3]. We supposed there that all coefficients $c_{j}, a_{j}, j=1, \ldots, n$, were independent random variables uniformly distributed on $[0,1]$, and the right-hand side was proportional to the number of variables, i.e., $b=\lambda n$. The main theorem in [3] stated that, if

$$
\lambda>\frac{1}{2}-\frac{t}{3},
$$


then both the primal and the dual greedy algorithms have an asymptotic tolerance $t$ (the exact definition is given at the beginning of the next section).

The assumption that the distribution of coefficients is the uniform one is rather traditional in the investigation of the average behaviour of algorithms. However, there exist real-world situations when this assumption is not justified (for example, there may be some "attraction zones" of the coefficient values). On the other hand, it is interesting to see to what extent the results from [3], obtained for the uniform distribution, can be generalized. Thus, deriving results about the average behaviour of greedy algorithms for arbitrary distributions is both of theoretical and practical interest. This generalization is the main subject of the present paper. Here we shall essentially reproduce the general structure of [3]; this will enable us to recognize some parallels as well as essential novel features.

\section{The main result}

We relax the main assumption made in [3] and suppose that the coefficients $c_{j}, a_{j}$, $j=1, . ., n$, are independent identically distributed random variables with an arbitrary distribution having the density $p(\cdot)$, which is almost everywhere positive. The corresponding distribution function will be denoted by $F(\cdot)$. The assumption about $b$ will be made a little later. We denote $z_{j}=c_{j} / a_{j}$. Let $z^{k}$ be the random variable equal to the $(n-k+1)$-th term of the variational series determined by the $n$-tuple of random variables $z_{1}, \ldots, z_{n}$, i.e., $z^{1} \geq z^{2} \geq \cdots \geq z^{n}$. We introduce now the random variables $c^{k}$, $a^{k}$ as follows. If $z^{k}=c_{q(k)} / a_{q(k)}$, we let

$$
c^{k}=c_{q(k)}, a^{k}=a_{q(k)} .
$$

We are interested in the behaviour of approximate algorithms for problems with $n$ variables when $n$ grows. Suppose that we are applying some approximate algorithm $\mathcal{A}$ to a combinatorial optimization problem. We denote this algorithm by $\mathcal{A}_{n}$ to stress the dependence on the number of variables. We say that the algorithm $\mathcal{A}_{n}$ has the asymptotical tolerance $t$ if

$$
\mathbf{P}\left(f^{*}-f^{\mathcal{A}_{n}} \leq t\right) \underset{n \rightarrow \infty}{\longrightarrow} 1,
$$

where $f^{*}$ is the optimal value, $t>0$.

This definition is rather general. Further we shall consider the knapsack problem (1), and $\mathcal{A}_{n}$ will be the greedy algorithm.

As in [3], we define the following events (the notation was given in the Introduction):

$$
\begin{aligned}
& A^{n}: f^{L R}-f^{D G} \leq t \\
& B_{k}^{n}: x^{L R}=(1, \ldots, 1, \alpha, 0, \ldots, 0), \text { where the component } \alpha \in[0,1) \text { stands on the place } \\
& k, k=1, \ldots, n . \\
& B_{n+1}^{n}: x^{L R}=(1, \ldots, 1)
\end{aligned}
$$


$C_{k}^{n}: c^{k} \leq t, k=1, \ldots, n$.

We formulate now two conditions.

C o n d i t i o n $1 . \sum_{k=N}^{n} \mathbf{P}\left(\bar{C}_{k}^{n}\right) \underset{n \rightarrow \infty}{\longrightarrow} 0$.

C o n d i t i o n 2. $\sum_{k=N}^{n+1} \mathbf{P}\left(B_{k}^{n}\right) \underset{n \rightarrow \infty}{\longrightarrow} 1$.

Now we choose a $t^{\prime}<t$ and denote $N=c n$, where $c=1-G\left(t^{\prime}\right)$ (the function $G(\cdot)$ is defined by the formula (11) in Section 3$)$.

Denote $a^{1}+\cdots+a^{N}=A^{N}$. It will be proved later that the expectation $\mathbf{E}\left(A^{N}\right)$ of $A^{N}$ is given by

$$
\mathbf{E}\left(A^{N}\right)=n \int_{0}^{1} \alpha p(\alpha) \int_{\alpha t^{\prime}}^{1} p(z) d z d \alpha+o(n) .
$$

Evidently, for $t^{\prime}<t$ we have

$$
\int_{0}^{1} \alpha p(\alpha) \int_{\alpha t^{\prime}}^{1} p(z) d z d \alpha>\int_{0}^{1} \alpha p(\alpha) \int_{\alpha t}^{1} p(z) d z d \alpha
$$

Now we claim that the following general result holds.

Theorem 1 Let $b=\lambda n$ where

$$
\lambda>\int_{0}^{1} \alpha p(\alpha) \int_{\alpha t}^{1} p(z) d z d \alpha .
$$

Let for $N=$ cn (where $c$ is defined above) the Conditions 1 and 2 hold. Then the primal and the dual greedy algorithms have the asymptotical tolerance $t$.

In [3] the following auxiliary results were proved.

Lemma 1 The following implication holds

$$
A^{n} \supset \sum_{k=N}^{n} B_{k}^{n} C_{k}^{n}+B_{n+1}^{n} .
$$

Lemma 2 The following inequalities hold

$$
\mathbf{P}\left(A^{n}\right) \geq \sum_{k=N}^{n} \mathbf{P}\left(B_{k}^{n} C_{k}^{n}\right) \geq \sum_{k=N}^{n}\left[\mathbf{P}\left(B_{k}^{n}\right)-\mathbf{P}\left(\bar{C}_{k}^{n}\right)\right] .
$$

In view of the representation of $\mathbf{E}\left(A^{N}\right)$ given above and Lemma 2, the proof of Theorem 1 can be given along the same lines as in [3].

In the next section we prove the validity of Condition 1 . Before doing that, we state a necessary condition for its fulfillment. This condition is evident: for all $y>0$ we must have

$$
\int_{0}^{y} p(x) d x>0 \text {. }
$$


Another important remark is the following. It can be seen from the proof that the density $p(\cdot)$ must have a bounded carrier (without loss of generality we can take $[0,1]$ ). We shall suppose that the density is almost everywhere positive on $[0,1]$.

The main goal of this paper is the proof of Condition 1 and the derivation of $\mathbf{E}\left(A^{N}\right)$. The analysis of requirements on $p(\cdot)$ that are necessary for the fulfillment of Condition 2 will be the subject of a subsequent paper. We are obliged to B. Bank [7] for suggesting some general ideas, which may be useful for deriving these requirements. (In [3] it was shown that for the uniform case, Condition 2 holds).

\section{The proof of Condition 1}

First of all, we find the main probabilistic characteristics of the random variables $c^{j}, a^{j}$ introduced at the beginning of Section 2. The values (realizations) of the random variables $c^{j}, a^{j}$ will be denoted by $\gamma_{j}, \alpha_{j}$ respectively.

The main role in the subsequent considerations belongs to the function

$$
G(z)=\mathbf{P}\left(z_{j} \leq z\right)
$$

which is the distribution function of the ratios. Now we give an explicit representation of this function $(F(\cdot)$ is the underlying distribution function).

Lemma 3 The following representation holds

$$
G(z)= \begin{cases}\int_{0}^{1} F(z x) p(x) d x & z \leq 1 \\ 1-\int_{0}^{1} F\left(\frac{y}{z}\right) p(y) d y & z \geq 1 .\end{cases}
$$

P r o o f. We have from the definition of $G(z)$

$$
G(z)=\mathbf{P}\left(z_{j} \leq z\right)=\mathbf{P}\left(\frac{c_{j}}{a_{j}} \leq z\right)=\mathbf{P}\left(c_{j} \leq z a_{j}\right) .
$$

Note that the joint density of $c_{j}, a_{j}$ is $p\left(c_{j}\right) p\left(a_{j}\right)$ (due to independence). Now, using the same geometric argument as in [2], we see that for $z \leq 1$

$$
\mathbf{P}\left(c_{j} \leq a_{j} z\right)=\int_{0}^{1} \int_{0}^{z a_{j}} p\left(c_{j}\right) p\left(a_{j}\right) d c_{j} d a_{j}=\int_{0}^{1} F\left(z a_{j}\right) p\left(a_{j}\right) d a_{j} .
$$

On the other hand, for $z \geq 1$ we have

$$
\begin{aligned}
\mathbf{P}\left(c_{j} \leq a_{j} z\right) & =1-\int_{0}^{1} \int_{0}^{a_{j} / z} p\left(c_{j}\right) p\left(a_{j}\right) d c_{j} d a_{j}= \\
& =1-\int_{0}^{1} F\left(\frac{a_{j}}{z}\right) p\left(a_{j}\right) d a_{j} .
\end{aligned}
$$

This proves (11) (up to the notation).

The next Lemma describes some simple properties of the function $G(z)$. 
Lemma 4 The function $G(z)$ defined in (11) has the following properties:

1) the function $y=G(z)$ is monotone increasing;

2) there exists the inverse function $z=G^{-1}(y)$, which is also monotone increasing;

3) the function $G(z)$ is differentiable, and its derivative is given by

$$
G^{\prime}(z)= \begin{cases}\int_{0}^{1} x p(z x) p(x) d x & z \leq 1 \\ \frac{1}{z^{2}} \int_{0}^{1} y p\left(\frac{y}{z}\right) p(y) d y & z \geq 1 .\end{cases}
$$

4) $G(1)=\frac{1}{2}$.

Indeed, the assertions 1) and 3) are immediate consequences of the representation (11). In turn, 1) implies 2). As for 4), we have from (11) that $G(1)=1-G(1)$.

If we consider the probability distribution not on the segment $[0,1]$ but on the infinite ray $[0, \infty)$, then Lemma 1 will be formulated as

Lemma $3^{\prime}$ The following representation holds

$$
G(z)=\int_{0}^{\infty} F(z x) p(x) d x .
$$

As for the proof, it becomes even simpler.

Note that in this case there are some interesting effects that do not occur in the case of finite intervals on which the probability measures are defined. The corresponding fact will be formulated a little later.

Another important remark is that for the particular case of the uniform distribution $(F(y)=y, p(y)=1$ for $y \in[0,1])$ the representation (11) yields

$$
G(z)=\left\{\begin{array}{ll}
\frac{z}{2} & z \leq 1 \\
1-\frac{1}{2 z} & z \geq 1
\end{array},\right.
$$

which is exactly the formula (13) from [3]. Thus, there is a possibility of checking every step of our further considerations by taking the corresponding particular "uniform case" and comparing the intermediary calculations with those from [3]. The results of this "quality control" are sometimes mentioned in the text.

Now we proceed to the determination of the density $f_{j}\left(\gamma_{j}\right)$.

Lemma 5 The density $f_{j}\left(\gamma_{j}\right)$ of the random variable $c^{j}$ is

$$
f_{j}\left(\gamma_{j}\right)=n\left(\begin{array}{c}
n-1 \\
j-1
\end{array}\right) \gamma_{j} p\left(\gamma_{j}\right) \int_{0}^{\infty}[G(u)]^{n-j}[1-G(u)]^{j-1} p\left(\frac{\gamma_{j}}{u}\right) \frac{d u}{u^{2}} .
$$


P r o o f. Recall that we analyze here only the case of a finite probability measure carrier (namely, $[0,1]$ ); nevertheless, we begin with the general formula $\left(11^{\prime}\right)$, having in mind some future considerations of the infinite case. We have

$$
\mathbf{P}\left(z_{k} \leq \frac{\gamma_{j}}{\alpha_{j}}\right)=\int_{0}^{1} F\left(\alpha_{k} \frac{\gamma_{j}}{\alpha_{j}}\right) p\left(\alpha_{k}\right) d \alpha_{k}=G\left(\frac{\gamma_{j}}{\alpha_{j}}\right)
$$

Then, similarly to [3], the probability that (for a fixed $j$ ) some $j-1$ random variables will be $\geq \gamma_{j} / \alpha_{j}$ and the remaining ones will be $\leq \gamma_{j} / \alpha_{j}$ is

$$
\pi\left(\gamma_{j} / \alpha_{j}\right)=n\left(\begin{array}{c}
n-1 \\
j-1
\end{array}\right)\left[G\left(\frac{\gamma_{j}}{\alpha_{j}}\right)\right]^{n-j}\left[1-G\left(\frac{\gamma_{j}}{\alpha_{j}}\right)\right]^{j-1}
$$

The joint density $r\left(\alpha_{j}, \gamma_{j}\right)$ of the random variables $a^{j}, c^{j}$ is

$$
r\left(\alpha_{j}, \gamma_{j}\right)=\pi\left(\gamma_{j} / \alpha_{j}\right) p\left(\gamma_{j}\right) p\left(\alpha_{j}\right) .
$$

Then the density $f_{j}\left(\gamma_{j}\right)$ of the random variable $c^{j}$ is

$$
f_{j}\left(\gamma_{j}\right)=\int_{0}^{\infty} r\left(\alpha_{j}, \gamma_{j}\right) d \alpha_{j}
$$

or

$$
f_{j}\left(\gamma_{j}\right)=n\left(\begin{array}{c}
n-1 \\
j-1
\end{array}\right) p\left(\gamma_{j}\right) \int_{0}^{\infty}\left[G\left(\frac{\gamma_{j}}{\alpha_{j}}\right)\right]^{n-j}\left[1-G\left(\frac{\gamma_{j}}{\alpha_{j}}\right)\right]^{j-1} p\left(\alpha_{j}\right) d \alpha_{j} .
$$

Changing here the variables $\gamma_{j} / \alpha_{j}=u$ we immediately obtain

$$
f_{j}\left(\gamma_{j}\right)=n\left(\begin{array}{c}
n-1 \\
j-1
\end{array}\right) \gamma_{j} p\left(\gamma_{j}\right) \int_{0}^{\infty}[G(u)]^{n-j}[1-G(u)]^{j-1} p\left(\frac{\gamma_{j}}{u}\right) \frac{d u}{u^{2}} .
$$

The proof of Lemma 5 is complete.

Now we derive the distribution function $F_{j}(t)$ of the random variable $c^{j}$. We have

$$
F_{j}(t)=\int_{0}^{t} f_{j}\left(\gamma_{j}\right) d \gamma_{j}
$$

or, using (13),

$$
F_{j}(t)=n\left(\begin{array}{c}
n-1 \\
j-1
\end{array}\right) \int_{0}^{t} \int_{0}^{\infty}[G(u)]^{n-j}[1-G(u)]^{j-1} p\left(\gamma_{j}\right) p\left(\frac{\gamma_{j}}{u}\right) \frac{\gamma_{j}}{u^{2}} d u d \gamma_{j} .
$$

We denote

$$
I(u, t)=\int_{0}^{t} \gamma_{j} p\left(\gamma_{j}\right) p\left(\frac{\gamma_{j}}{u}\right) d \gamma_{j}
$$

Using (15) we can rewrite (14) as

$$
F_{j}(t)=n\left(\begin{array}{c}
n-1 \\
j-1
\end{array}\right) \int_{0}^{\infty}[G(u)]^{n-j}[1-G(u)]^{j-1} \frac{I(u, t)}{u^{2}} d u .
$$


Now we introduce new variables

$$
G(u)=y
$$

We have from (17)

$$
u=G^{-1}(y), \quad d u=\frac{d y}{G^{\prime}(u)}=\frac{d y}{G^{\prime}\left(G^{-1}(y)\right)} .
$$

Rewrite (16) using (17) and (18):

$$
F_{j}(t)=n\left(\begin{array}{c}
n-1 \\
j-1
\end{array}\right) \int_{0}^{1} y^{n-j}(1-y)^{j-1} \frac{I\left(G^{-1}(y), t\right)}{\left[G^{-1}(y)\right]^{2} G^{\prime}\left(G^{-1}(y)\right)} d y .
$$

Denote

$$
J(y, t)=\frac{I\left(G^{-1}(y), t\right)}{\left[G^{-1}(y)\right]^{2} G^{\prime}\left(G^{-1}(y)\right)} .
$$

Thus, using (17), we obtain

$$
J(G(u), t)=\frac{I(u, t)}{u^{2} G^{\prime}(u)} .
$$

Now we have to establish an intermediary result. This time its validity depends crucially on the assumption that the random variables are defined on $[0,1]$.

Lemma 6 For $u \leq t$ we have

$$
J(G(u), t)=1
$$

P r o o f. The assertion 3) of Lemma 4 yields an explicit representation for $G^{\prime}(u)$ :

$$
G^{\prime}(u)=\int_{0}^{1} x p(x) p(u x) d x .
$$

In (15) we define new variables $\gamma_{j} / u=z$. This yields

$$
I(u, t)=u^{2} \int_{0}^{\frac{t}{u}} z p(z) p(u z) d z .
$$

From (21) we have

$$
J(G(u), t)=\frac{u^{2} \int_{0}^{\frac{t}{u}} z p(z) p(u z) d z}{u^{2} \int_{0}^{1} x p(x) p(u x) d x} .
$$

The assumption that the probability measure carrier is $[0,1]$ implies that the integral in the nominator of (22) is actually from 0 to 1 , since $u \leq t$ implies $t / u \geq 1$, and for $1<z \leq t / u$ the density $p(z)=0$. Thus, the assertion of Lemma 6 is proved.

Note that for the carrier $[0, \infty)$ the ratio in (22) is greater than 1 , and this implies that the Condition 1 in this case does not hold.

The condition $u \leq t$ of Lemma 6 means that $G^{-1}(y) \leq t$ or $y \leq G(t)$. Thus Lemma 6 says that

$$
J(y, t)=1 \quad \text { for } \quad y \leq G(t) .
$$


Now we obtain from (19), (20), (21) and (23) that

$$
\begin{aligned}
F_{j}(t) & =n\left(\begin{array}{c}
n-1 \\
j-1
\end{array}\right) \int_{0}^{G(t)} y^{n-j}(1-y)^{j-1} d y+ \\
& +n\left(\begin{array}{c}
n-1 \\
j-1
\end{array}\right) \int_{G(t)}^{1} y^{n-j}(1-y)^{j-1} J(y, t) d y .
\end{aligned}
$$

We can summarize these considerations as

Lemma 7 The distribution function $F_{j}(t)$ of the random variable $c^{j}$ has the representation (24).

We proceed now to the proof of the main assertion of this section.

Theorem 2 Let $N=$ cn where $c>1-G(t)$. Then the Condition 1 is fulfilled.

P r o o f. We start with the representation (24) and consider its first summand

$$
\Phi_{j}(t)=n\left(\begin{array}{c}
n-1 \\
j-1
\end{array}\right) \int_{0}^{G(t)} y^{n-j}(1-y)^{j-1} d y
$$

The well-known formula from [6] states that

$$
B(k, n, p)=(n-k)\left(\begin{array}{l}
n \\
k
\end{array}\right) \int_{0}^{1-p} t^{n-k-1}(1-t)^{k} d t .
$$

Take $j=k+1$ in (25). This yields

$$
\Phi_{k+1}(t)=n\left(\begin{array}{c}
n-1 \\
k
\end{array}\right) \int_{0}^{G(t)} y^{n-k-1}(1-y)^{k} d y
$$

It is clear that

$$
n\left(\begin{array}{c}
n-1 \\
k
\end{array}\right)=(n-k)\left(\begin{array}{l}
n \\
k
\end{array}\right)
$$

Therefore

$$
\Phi_{k+1}(t)=(n-k)\left(\begin{array}{l}
n \\
k
\end{array}\right) \int_{0}^{G(t)} y^{n-k-1}(1-y)^{k} d y .
$$

We see that the right-hand side of (27) coincides with the right-hand side of (26) for $1-p=G(t)$. Therefore

$$
\Phi_{k+1}(t)=B(k, n, 1-G(t))=\sum_{\nu=0}^{k}\left(\begin{array}{l}
n \\
\nu
\end{array}\right)[1-G(t)]^{\nu}[G(t)]^{n-\nu} .
$$

Now, as said before, for the proof of Condition 1 we put $N=c n$ and, together with the proof of this condition, we findsimultaneously for which values of $c$ this is satisfied. 
We rewrite (24) taking into account (28):

$$
F_{k+1}(t)=\sum_{\nu=0}^{k}\left(\begin{array}{l}
n \\
\nu
\end{array}\right)[1-G(t)]^{\nu}[G(t)]^{n-\nu}+n\left(\begin{array}{c}
n-1 \\
k
\end{array}\right) \int_{G(t)}^{1} y^{n-k-1}(1-y)^{k} J(y, t) d y .
$$

We have

$$
\begin{aligned}
\mathbf{P}\left(\bar{C}_{k+1}^{n}\right) & =1-F_{k+1}(t)= \\
& =\sum_{\nu=k+1}^{n}\left(\begin{array}{l}
n \\
\nu
\end{array}\right)[1-G(t)]^{\nu}[G(t)]^{n-\nu}- \\
& -n\left(\begin{array}{c}
n-1 \\
k
\end{array}\right) \int_{G(t)}^{1} y^{n-k-1}(1-y)^{k} J(y, t) d y \leq \\
& \leq \sum_{\nu=k+1}^{n}\left(\begin{array}{l}
n \\
\nu
\end{array}\right)[1-G(t)]^{\nu}[G(t)]^{n-\nu} .
\end{aligned}
$$

We take, as said before, $N=c n$ and estimate the sum

$$
\sum_{k=N}^{n} \mathbf{P}\left(\bar{C}_{k}^{n}\right) \leq \sum_{k=N}^{n} \sum_{\nu=k+1}^{n}\left(\begin{array}{l}
n \\
\nu
\end{array}\right)[1-G(t)]^{\nu}[G(t)]^{n-\nu} .
$$

A simple transformation of the double sum in the right-hand side yields

$$
\sum_{k=N}^{n} \sum_{\nu=k+1}^{n} a_{\nu}=\sum_{\nu=N+1}^{n}(\nu-N) a_{\nu}
$$

Thus, we have to estimate the sum (which we rewrite changing the summation index)

$$
\sum_{j=N+1}^{n}(j-N)\left(\begin{array}{l}
n \\
j
\end{array}\right)[1-G(t)]^{j}[G(t)]^{n-j} .
$$

Let

$$
b_{j}=\left(\begin{array}{c}
n \\
j
\end{array}\right)[1-G(t)]^{j}[G(t)]^{n-j} .
$$

The well-known calculation (cf.[6])

$$
\frac{b_{j}}{b_{j-1}}=\frac{(n-j+1)[1-G(t)]}{j G(t)}=1+\frac{(n+1)[1-G(t)]-j}{j G(t)}
$$

shows that the sequence $\left\{b_{j}\right\}$ decreases when $b_{j} / b_{j-1}<1$ or

$$
j>(n+1)[1-G(t)] .
$$

The first term in the sum (30) is maximal if

$$
N+1>(n+1)[1-G(t)] .
$$


We took $N=c n, c<1$; therefore

$$
c n+1>(n+1)[1-G(t)] .
$$

The last inequality yields

$$
c>1-G(t)\left(1+\frac{1}{n}\right) .
$$

Let the inequality (31) be satisfied. We carry out the maximal summand in the sum (29):

$$
\begin{aligned}
\sum_{j=N+1}^{n} \mathbf{P}\left(\bar{C}_{j}^{n}\right) & \leq\left(\begin{array}{c}
n \\
N+1
\end{array}\right)[1-G(t)]^{N+1}\left[G(t)^{n-N-1} \times\right. \\
& \times\left(1+2 \frac{b_{N+2}}{b_{N+1}}+3 \frac{b_{N+3}}{b_{N+1}}+\cdots+(n-N) \frac{b_{n}}{b_{N+1}}\right) .
\end{aligned}
$$

We calculate the ratio

$$
\frac{b_{N+2}}{b_{N+1}}=\frac{(n-N-1)[1-G(t)]}{(N+2) G(t)}=\alpha .
$$

It is easy to show that for $k>2$

$$
\frac{b_{N+k}}{b_{N+1}} \leq \alpha^{k}
$$

However, $\alpha$, as it is defined in (32), depends on $n$. Substituting $N=c n$ in (32) we obtain

$$
\alpha=\frac{(n-c n-1)[1-G(t)]}{(c n+2) G(t)}=\frac{\left(1-c-\frac{1}{n}\right)[1-G(t)]}{\left(c+\frac{2}{n}\right) G(t)} \leq \frac{(1-c)[1-G(t)]}{c G(t)}=\beta .
$$

We see that $\alpha=\alpha(n) \rightarrow \beta$ when $n \rightarrow \infty$. Therefore

$$
\sum_{k=N+1}^{n} \mathbf{P}\left(\bar{C}_{k}^{n}\right)<\left(\begin{array}{c}
n \\
N+1
\end{array}\right)[1-G(t)]^{N+1}[G(t)]^{n-N-1}\left(1+2 \beta+3 \beta^{2}+\ldots\right) .
$$

Obviously,

$$
1+2 \beta+3 \beta^{2}+\cdots=\left(\beta+\beta^{2}+\beta^{3}+\cdots\right)^{\prime}=\left(\frac{\beta}{1-\beta}\right)^{\prime}=\frac{1}{(1-\beta)^{2}} .
$$

For the validity of this we must have $\beta<1$, that is

$$
(1-c)[1-G(t)]<c G(t)
$$

or

$$
c>1-G(t) .
$$

This condition is a little bit stronger than (31). Thus, for the convergence we must impose the condition (34). Passing in the inequality (33) to the limit yields

$$
\lim _{n \rightarrow \infty} \sum_{k=N+1}^{n} \mathbf{P}\left(\bar{C}_{k}^{n}\right) \leq \lim _{n \rightarrow \infty}\left(\begin{array}{c}
n \\
N+1
\end{array}\right)[1-G(t)]^{N+1}[G(t)]^{n-N-1} \frac{1}{(1-\beta)^{2}} .
$$

We see that the right-hand side of this inequality tends to zero when $n \rightarrow \infty$. The proof of Theorem 2 is complete. 


\section{The expected value of $a^{1}+\cdots+a^{N}$}

We take again $N$ as it was defined in the previous section:

$$
N=n\left(1-G\left(t^{\prime}\right)\right)
$$

where $t^{\prime}<t$ (see also Section 2). We introduce the notation

$$
A^{N}=a^{1}+\cdots+a^{N} .
$$

Our main goal here is the computation of the expected value $\mathbf{E}\left(A^{N}\right)$. For this we need firstly the density $g_{j}\left(\alpha_{j}\right)$ of the random variable $a^{j}$.

First of all we return to the representation (13) for the density $f_{j}\left(\gamma_{j}\right)$ of the random variable $c^{j}$ given in Lemma 5:

$$
f_{j}\left(\gamma_{j}\right)=n\left(\begin{array}{c}
n-1 \\
j-1
\end{array}\right) \gamma_{j} p\left(\gamma_{j}\right) \int_{0}^{\infty}[G(u)]^{n-j}[1-G(u)]^{j-1} p\left(\frac{\gamma_{j}}{u}\right) \frac{d u}{u^{2}} .
$$

Note that this integral is non-zero only for $u \geq \gamma_{j}$, since for $u<\gamma_{j}$ we have $\gamma_{j} / u>1$, and the density $p\left(\gamma_{j} / u\right)=0$. Taking this into account we rewrite (13), splitting it into two summands for convenience:

$$
\begin{aligned}
f_{j}\left(\gamma_{j}\right) & =n\left(\begin{array}{c}
n-1 \\
j-1
\end{array}\right)\left[\gamma_{j} p\left(\gamma_{j}\right) \int_{\gamma_{j}}^{1}[G(u)]^{n-j}[1-G(u)]^{j-1} p\left(\frac{\gamma_{j}}{u}\right) \frac{d u}{u^{2}}+\right. \\
& \left.+\gamma_{j} p\left(\gamma_{j}\right) \int_{1}^{\infty}[G(u)]^{n-j}[1-G(u)]^{j-1} p\left(\frac{\gamma_{j}}{u}\right) \frac{d u}{u^{2}}\right] .
\end{aligned}
$$

Now we apply the change of variables (17) (see also (18)) to $\left(13^{\prime}\right)$. Note here that $G(1)=\frac{1}{2}$ and that for $u=\infty$ we have $y=1$. This yields

$$
\begin{aligned}
f_{j}\left(\gamma_{j}\right) & =n\left(\begin{array}{c}
n-1 \\
j-1
\end{array}\right)\left[\gamma_{j} p\left(\gamma_{j}\right) \int_{G\left(\gamma_{j}\right)}^{\frac{1}{2}} y^{n-j}(1-y)^{j-1} p\left(\frac{\gamma_{j}}{G^{-1}(y)}\right) \frac{1}{\left[G^{-1}(y)\right]^{2}} \frac{d y}{G^{\prime}\left(G^{-1}(y)\right)}+\right. \\
& \left.+\gamma_{j} p\left(\gamma_{j}\right) \int_{\frac{1}{2}}^{1} y^{n-j}(1-y)^{j-1} p\left(\frac{\gamma_{j}}{G^{-1}(y)}\right) \frac{1}{\left[G^{-1}(y)\right]^{2}} \frac{d y}{G^{\prime}\left(G^{-1}(y)\right)}\right] .
\end{aligned}
$$

$\mathrm{R}$ e $\mathrm{m}$ a $\mathrm{r} \mathrm{k}$. As we noted above, it is possible to check every step by considering the particular case of the uniform distribution. For this case we have (cf. (12))

$$
G(u)=y= \begin{cases}\frac{u}{2} & u \leq 1 \\ 1-\frac{1}{2 u} & u \geq 1 .\end{cases}
$$

From this we easily find all the functions we need:

$$
u=G^{-1}(y)= \begin{cases}2 y & y \leq \frac{1}{2} \\ \frac{1}{2(1-y)} & y \geq \frac{1}{2}\end{cases}
$$




$$
\begin{aligned}
G^{\prime}(u) & = \begin{cases}\frac{1}{2} & u \leq 1 \\
\frac{1}{2 u^{2}} & u \geq 1\end{cases} \\
G^{\prime}\left(G^{-1}(y)\right) & = \begin{cases}\frac{1}{2} & y \leq \frac{1}{2} \\
2(1-y)^{2} & y \geq \frac{1}{2}\end{cases}
\end{aligned}
$$

Substituting these in $\left(13^{\prime \prime}\right)$ yields

$$
f_{j}\left(\gamma_{j}\right)=n\left(\begin{array}{c}
n-1 \\
j-1
\end{array}\right)\left[\frac{\gamma_{j}}{2} \int_{\frac{\gamma_{j}}{2}}^{\frac{1}{2}} y^{n-j-2}(1-y)^{j-1} d y+2 \gamma_{j} \int_{0}^{\frac{1}{2}} z^{j-1}(1-z)^{n-j} d z\right],
$$

which is exactly the density for the uniform distribution (cf. the formula (12) in [3]).

After these preliminaries we can formulate the following result.

Lemma 8 The density $g_{j}\left(\alpha_{j}\right)$ of the random variable $a^{j}$ has the form

$$
\begin{aligned}
g_{j}\left(\alpha_{j}\right) & =n\left(\begin{array}{c}
n-1 \\
j-1
\end{array}\right)\left[\alpha_{j} p\left(\alpha_{j}\right) \int_{G\left(\alpha_{j}\right)}^{\frac{1}{2}} y^{j-1}(1-y)^{n-j} p\left(\frac{\alpha_{j}}{G^{-1}(y)}\right) \frac{1}{\left[G^{-1}(y)\right]^{2}} \frac{d y}{G^{\prime}\left(G^{-1}(y)\right)}+\right. \\
& \left.+\alpha_{j} p\left(\alpha_{j}\right) \int_{\frac{1}{2}}^{1} y^{j}(1-y)^{n-j} p\left(\frac{\alpha_{j}}{G^{-1}(y)}\right) \frac{1}{\left[G^{-1}(y)\right]^{2}} \frac{d y}{G^{\prime}\left(G^{-1}(y)\right)}\right]
\end{aligned}
$$

P r o o f. For obtaining $g_{j}\left(\alpha_{j}\right)$ we could, similarly to the proof of Lemma 5, integrate the joint density $r\left(\alpha_{j}, \gamma_{j}\right)$ of $a^{j}, c^{j}$ over $\gamma_{j}$. A simpler reasoning consists in the following (cf. [3]). The inequalities

$$
\frac{c^{1}}{a^{1}} \geq \frac{c^{2}}{a^{2}} \geq \cdots \geq \frac{c^{n}}{a^{n}}
$$

are equivalent to

$$
\frac{a^{n}}{c^{n}} \geq \frac{a^{n-1}}{c^{n-1}} \geq \cdots \geq \frac{a^{1}}{c^{1}} .
$$

Therefore $a^{j}$ has the same density as $c^{n-j+1}$. Substituting $\alpha_{j}$ for $\gamma_{j}$ in any representation of $f_{j}\left(\gamma_{j}\right)\left((13),\left(13^{\prime}\right)\right.$ or $\left.\left(13^{\prime \prime}\right)\right)$ and replacing $j$ in the exponents by $n-j+1$, we obtain the desired density.

Now we proceed to the calculation of $\mathbf{E}\left(A^{N}\right)$. For this, the expected value of $a^{j}$ is necessary. We have from (35)

$$
\begin{aligned}
\mathbf{E}\left(a^{j}\right) & =n\left(\begin{array}{c}
n-1 \\
j-1
\end{array}\right)\left[\int_{0}^{1} \alpha_{j}^{2} p\left(\alpha_{j}\right) \int_{G\left(\alpha_{j}\right)}^{\frac{1}{2}} y^{j-1}(1-y)^{n-j} p\left(\frac{\alpha_{j}}{G^{-1}(y)}\right) \frac{1}{\left[G^{-1}(y)\right]^{2}} \frac{d y}{G^{\prime}\left(G^{-1}(y)\right)} d \alpha_{j}+\right. \\
& \left.+\int_{0}^{1} \alpha_{j}^{2} p\left(\alpha_{j}\right) \int_{\frac{1}{2}}^{1} y^{j-1}(1-y)^{n-j} p\left(\frac{\alpha_{j}}{G^{-1}(y)}\right) \frac{1}{\left[G^{-1}(y)\right]^{2}} \frac{d y}{G^{\prime}\left(G^{-1}(y)\right)} d \alpha_{j}\right]
\end{aligned}
$$


We change the integration order in (35). This yields

$$
\begin{aligned}
\mathbf{E}\left(a^{j}\right) & =n\left(\begin{array}{c}
n-1 \\
j-1
\end{array}\right)\left[\int_{0}^{\frac{1}{2}} y^{j-1}(1-y)^{n-j} \frac{1}{\left[G^{-1}(y)\right]^{2}} \int_{0}^{G^{-1}(y)} \alpha_{j}^{2} p\left(\alpha_{j}\right) p\left(\frac{\alpha_{j}}{G^{-1}(y)}\right) d \alpha_{j} d y+\right. \\
& \left.+\int_{\frac{1}{2}}^{1} y^{j-1}(1-y)^{n-j} \frac{1}{\left[G^{-1}(y)\right]^{2}} \int_{0}^{1} \alpha_{j}^{2} p\left(\alpha_{j}\right) p\left(\frac{\alpha_{j}}{G^{-1}(y)}\right) d \alpha_{j} d y\right] .
\end{aligned}
$$

Now we are prepared to prove the main theorem of this section.

Theorem 3 The expected value of $A^{N}$ is

$$
\mathbf{E}\left(A^{N}\right)=n \int_{0}^{1} \alpha p(\alpha) \int_{\alpha t^{\prime}}^{1} p(z) d z d \alpha+o(n) .
$$

P r o o f. We have from (37) that

$$
\begin{aligned}
\mathbf{E}\left(A^{N}\right) & =n\left[\int_{0}^{\frac{1}{2}} \sum_{j=1}^{N}\left(\begin{array}{c}
n-1 \\
j-1
\end{array}\right) y^{j-1}(1-y)^{n-j} \frac{1}{\left[G^{-1}(y)\right]^{2}} \frac{1}{G^{\prime}\left(G^{-1}(y)\right)} \times\right. \\
& \times \int_{0}^{G^{-1}(y)} \alpha_{j}^{2} p\left(\alpha_{j}\right) p\left(\frac{\alpha_{j}}{G^{-1}(y)}\right) d \alpha_{j} d y+ \\
& +\int_{\frac{1}{2}}^{1} \sum_{j=1}^{N}\left(\begin{array}{c}
n-1 \\
j-1
\end{array}\right) y^{j-1}(1-y)^{n-j} \frac{1}{\left[G^{-1}(y)\right]^{2}} \frac{1}{G^{\prime}\left(G^{-1}(y)\right)} \times \\
& \left.\times \int_{0}^{1} \alpha_{j}^{2} p\left(\alpha_{j}\right) p\left(\frac{\alpha_{j}}{G^{-1}(y)}\right) d \alpha_{j} d y\right] .
\end{aligned}
$$

We rewrite this equality setting $j-1=k$ :

$$
\begin{aligned}
\mathbf{E}\left(A^{N}\right) & =n\left[\int_{0}^{\frac{1}{2}} \sum_{k=0}^{N-1}\left(\begin{array}{c}
n-1 \\
k
\end{array}\right) y^{k}(1-y)^{n-k-1} \frac{1}{\left[G^{-1}(y)\right]^{2}} \frac{1}{G^{\prime}\left(G^{-1}(y)\right)} \times\right. \\
& \times \int_{0}^{G^{-1}(y)} \alpha_{j}^{2} p\left(\alpha_{j}\right) p\left(\frac{\alpha_{j}}{G^{-1}(y)}\right) d \alpha_{j} d y+ \\
& +\int_{\frac{1}{2}}^{1} \sum_{k=0}^{N-1}\left(\begin{array}{c}
n-1 \\
k
\end{array}\right) y^{k}(1-y)^{n-k-1} \frac{1}{\left[G^{-1}(y)\right]^{2}} \frac{1}{G^{\prime}\left(G^{-1}(y)\right)} \times \\
& \left.\times \int_{0}^{1} \alpha_{j}^{2} p\left(\alpha_{j}\right) p\left(\frac{\alpha_{j}}{G^{-1}(y)}\right) d \alpha_{j} d y\right] .
\end{aligned}
$$

Now we apply the "zero-one" lemma (cf. Lemma 12 from [3]) to (39). It states the following. Denote

$$
B_{n}=B\left(m_{n}+\mu, n-1,1-x\right)=\sum_{j=0}^{m_{n}+\mu}\left(\begin{array}{c}
n-1 \\
j
\end{array}\right)(1-x)^{j} x^{n-j-1} .
$$

Then two assertions hold: 
1) Let $m_{n} \geq \lambda n$ where $\lambda>1-x$. Then $B_{n} \rightarrow 1$ if $n \rightarrow \infty$;

2) Let $m_{n}<\lambda n$ where $\lambda<1-x$. Then $B_{n} \rightarrow 0$ if $n \rightarrow \infty$;

We take, as said before, $N=c n$ where $c=1-G\left(t^{\prime}\right), t^{\prime}<t$. We see that in our case $B_{n} \rightarrow 1$ for $y<1-G\left(t^{\prime}\right)$. It follows that in the first integral from (39) the binomial sum tends to 1 in the entire domain $\left[0, \frac{1}{2}\right]$, and in the second integral - in the domain $\left[\frac{1}{2}, 1-G\left(t^{\prime}\right)\right]$. Taking this into account we rewrite (39), writing $\alpha$ instead of $\alpha_{j}$ for simplicity (note that the conditions of Lebesgue's Bounded Convergence Theorem are fulfilled). This yields

$$
\begin{aligned}
\mathbf{E}\left(A^{N}\right) & =n\left[\int_{0}^{\frac{1}{2}} \frac{1}{\left[G^{-1}(y)\right]^{2}} \frac{1}{G^{\prime}\left(G^{-1}(y)\right)} \times\right. \\
& \times \int_{0}^{G^{-1}(y)} \alpha^{2} p(\alpha) p\left(\frac{\alpha}{G^{-1}(y)}\right) d \alpha d y+ \\
& +\int_{\frac{1}{2}}^{1-G\left(t^{\prime}\right)} \frac{1}{\left[G^{-1}(y)\right]^{2}} \frac{1}{G^{\prime}\left(G^{-1}(y)\right)} \times \\
& \left.\times \int_{0}^{1} \alpha^{2} p(\alpha) p\left(\frac{\alpha}{G^{-1}(y)}\right) d \alpha d y\right]+o(n) .
\end{aligned}
$$

Note that, for the uniform distribution, (40) yields

$$
\mathbf{E}\left(A^{N}\right)=n\left(\frac{1}{2}-\frac{t^{\prime}}{3}\right)+o(n),
$$

i.e., exactly the same as in [3]. We change the integration order in (40):

$$
\begin{aligned}
\mathbf{E}\left(A^{N}\right) & =n\left[\int_{0}^{1} \alpha^{2} p(\alpha) \int_{G(\alpha)}^{\frac{1}{2}} p\left(\frac{\alpha}{G^{-1}(y)}\right) \frac{1}{\left[G^{-1}(y)\right]^{2}} \frac{1}{G^{\prime}\left(g^{-1}(y)\right)} d y d \alpha+\right. \\
& \left.+\int_{0}^{1} \alpha^{2} p(\alpha) \int_{\frac{1}{2}}^{1-G\left(t^{\prime}\right)} p\left(\frac{\alpha}{G^{-1}(y)}\right) \frac{1}{\left[G^{-1}(y)\right]^{2}} \frac{1}{G^{\prime}\left(G^{-1}(y)\right)} d y d \alpha\right]+o(n) .
\end{aligned}
$$

Now we return to the original variables $u=G^{-1}(y)$. For $y=1-G\left(t^{\prime}\right)$ we have $u=$ $G^{-1}\left(1-G\left(t^{\prime}\right)\right)$. Thus we have, after obvious simplifications,

$$
\begin{aligned}
\mathbf{E}\left(A^{N}\right) & =n\left[\int_{0}^{1} \alpha^{2} p(\alpha) \int_{\alpha}^{1} p\left(\frac{\alpha}{u}\right) \frac{d u}{u^{2}} d \alpha+\right. \\
& \left.+\int_{0}^{1} \alpha^{2} p(\alpha) \int_{1}^{G^{-1}\left(1-G\left(t^{\prime}\right)\right)} p\left(\frac{\alpha}{u}\right) \frac{d u}{u^{2}} d \alpha\right]+o(n) .
\end{aligned}
$$

We interrupt the proof of Theorem 3 for finding the upper integration limit in the second integral in (41).

Lemma 9 For all $t \leq 1$ we have

$$
G^{-1}(1-G(t))=\frac{1}{t}
$$


P r o o f. Denote

$$
G^{-1}(1-G(t))=z(t)
$$

Earlier (Lemma 3) we had the integral representation for $G(t)$ :

$$
G(t)= \begin{cases}\int_{0}^{1} F(t x) p(x) d x & t \leq 1 \\ 1-\int_{0}^{1} F\left(\frac{x}{t}\right) p(x) d x & t \geq 1 .\end{cases}
$$

Besides, we have shown (Lemma 4) that $G(1)=\frac{1}{2}$ which implies that $G^{-1}\left(\frac{1}{2}\right)=1$ and, from the monotonicity of $G^{-1}$ (Lemma 4$), G^{-1}(y) \geq 1$ for $y \geq \frac{1}{2}$. At the same time,

$$
\begin{gathered}
G(t) \leq \frac{1}{2} \quad t \leq 1 \\
1-G(t) \geq \frac{1}{2} \quad t \geq 1 .
\end{gathered}
$$

Therefore

$$
z(t)=G^{-1}(1-G(t)) \geq 1
$$

From (42) we have

$$
1-G(t)=G(z(t)) .
$$

Now the representation (43) yields

$$
1-\int_{0}^{1} F(t x) p(x) d x=1-\int_{0}^{1} F\left(\frac{x}{z(t)}\right) p(x) d x
$$

or

$$
\int_{0}^{1} F(t x) p(x) d x=\int_{0}^{1} F\left(\frac{x}{z(t)}\right) p(x) d x .
$$

Denote the left-hand side of $(44)$ by $\varphi(t)$. We have

$$
\varphi^{\prime}(t)=t \int_{0}^{1} p(t x) p(x) d x .
$$

Thus, $\varphi^{\prime}(t)>0$ for all $t>0$, that is, $\varphi(t)$ is a strictly increasing function. Therefore,

$$
t=\frac{1}{z(t)}
$$

or

$$
z(t)=\frac{1}{t}
$$

The proof of Lemma 9 is complete. 
We return to the proof of Theorem 3. Rewrite the expression (41) for $\mathbf{E}\left(A^{N}\right)$ uniting both integrals:

$$
\mathbf{E}\left(A^{N}\right)=n \int_{0}^{1} \alpha^{2} p(\alpha) \int_{\alpha}^{\frac{1}{t^{\prime}}} p\left(\frac{\alpha}{u}\right) \frac{d u}{u^{2}} d \alpha+o(n) .
$$

The final step is changing the variables in (45) $\alpha / u=z$. This yields

$$
\mathbf{E}\left(A^{N}\right)=n \int_{0}^{1} \alpha p(\alpha) \int_{\alpha t^{\prime}}^{1} p(z) d z d \alpha+o(n)
$$

The proof of Theorem 3 is complete.

Note that for the case of the uniform distribution we obtain again

$$
\mathbf{E}\left(A^{N}\right)=n\left(\frac{1}{2}-\frac{t^{\prime}}{3}\right)+o(n)
$$

(cf. the formula (29) in [3]).

\section{Acknowledgements}

The authors are indebted to the Deutsche Forschungsgemeinschaft (DFG) for the support which made the completion of this research possible. We are obliged to the participants of the seminars at the Humboldt-Universität zu Berlin, Otto-von-Guericke Universität Magdeburg, especially to B. Bank, J. Guddat, H. Hollatz and B. Kummer for valuable discussions. The second author also thanks the Institut für Mathematik der HumboldtUniversität zu Berlin for providing excellent working conditions.

\section{References}

[1 ] Korte, B. Kombinatorische Optimierung and algorithmische Prinzipien. In: W.Krelle, ed. Ökonomische Prognose-, Entscheidungs- und Gleichgewichtsmodelle, VCH Verlagsgesellschaft, Weinheim, 1986, 286-341.

[2 ] Diubin, G.N. and A.A.Korbut. Primal and dual greedy algorithms for the knapsack problem: Worst-case vs. average behaviour. SFB Report 121, Institute of Mathematics, Technical University Graz, Austria, 1997.

[3 ] Diubin, G.N. and A.A.Korbut. On the average behaviour of greedy algorithms for the knapsack problem. Preprint 99-14, Humboldt-Universität zu Berlin, Mathematisch-Naturwissenschaftliche Fakultät II, Institut für Mathematik, 1999.

[4 ] Korbut, A.A. The characterization of dual greedy algorithms for independence systems (in Russian). In: Ekonomiko-matematicheskie issledovania: matematicheskoe modelirovanie i informazionnie technologii. Nauka, St.Petersburg, 2000, 42-51. 
[5 ] Diubin, G.N. and A.A.Korbut. On the average behaviour of primal and dual greedy algorithms for the knapsack problem.In: U.Zimmermann et al., eds., Operations Research Proceedings 1996, Springer, Berlin, 1997, 55-60.

[6 ] Feller, W. An introduction to probability theory and its applications., vol. I, 2nd ed., J.Wiley and Sons, New York, 1965.

[7 ] Bank, B. Private communication. November 2000 